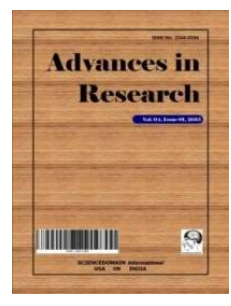

Advances in Research

6(6): 1-9, 2016, Article no.AIR.24580

ISSN: 2348-0394, NLM ID: 101666096

SCIENCEDOMAIN international

www.sciencedomain.org

\title{
A High-p/Low-p Procedure to Improve Recall Memory in Elderly Patients with Mild to Moderate Cognitive Impairment
}

\author{
Dawn A. Seefeldt ${ }^{1}$, Daniel Houlihan ${ }^{1^{*}}$ and Jeffrey A. Buchanan ${ }^{1}$ \\ ${ }^{1}$ Minnesota State University, Mankato, USA.
}

Authors' contributions

All authors participated in the design of the study. Authors JAB and DAS assessed participants. Author DAS collected and analyzed the data. Authors DAS and JAB wrote the initial draft and author $D H$ made significant editorial contributions. All authors read and approved the final manuscript.

Article Information

DOI: $10.9734 /$ AIR/2016/24580

Editor(s):

(1) Chung-Yao Hsu, Division of Epilepsy and Sleep Disorders, Department of Neurology, College of Medicine, Kaohsiung Medical University, Taiwan. (2) Jinyong Peng, Professor, College of Pharmacy, Dalian Medical University, Dalian, China.

Reviewers

(1) Jorge Alfredo Herrera Pino, Florida International University, Miami, USA. (2) Maria Gabriela Ramos Ferreira, University of the Region of Joinville, Brazil. (3) Elaine Cristina Zachi, University of Sao Paulo, Brazil. (4) Thiara Joanna Peçanha da Cruz, Minas Gerais Federal University, Brazil. Complete Peer review History: http://sciencedomain.org/review-history/13828

Method Article

Received $26^{\text {th }}$ January 2016 Accepted $10^{\text {th }}$ March 2016 Published 24 ${ }^{\text {th }}$ March 2016

\begin{abstract}
The objective of this study was to demonstrate the effectiveness of implementing two types of highprobability of naming/low-probability of naming procedures to improve object recall memory in elderly individuals with cognitive impairment. The procedures involved the use of directives for recall compliance with a demonstrated high rate of compliance followed by embedded directives with a demonstrated low rate of recall. Two elderly subjects in states of cognitive decline were used in this demonstration. Results indicated positive outcomes with both procedures. Recall-based interventions were more effective than recognition-based procedures.
\end{abstract}

Keywords: Dementia; memory enhancement; cognitive rehabilitation; high-probability of occurrence of naming sequences; high-p; low-p. 


\section{INTRODUTION}

Cognitive impairment in older adulthood affects millions and is projected to impact an estimated 115.4 million by 2050 [1]. Developing nonpharmacological interventions that slow memory loss and maintain autonomy is imperative [2].

Memory types vary and are differentially impacted by the effects of normal aging and conditions causing dementia (e.g., Alzheimer's disease). Recall memory involves retrieving previously learned information without presentation of answer choices, such as remembering a person's name [3]. Cued recall involves providing a prompt to guide recall [3], such as a picture followed by a categorical cue (e.g., "What animals did you see?"). Recognition differs in that the correct answer is embedded within presented choices with more environmental support [3,4]. For instance, "Is his name Bob, Tom, George, or Sam?" With aging, recognition and recall memory differentially decline with recall diminishing earliest [5].

"High-p" (high-probability of naming) and "lowp"(low-probability of naming) procedures were implemented in this study in an effort to improve object naming in elderly individuals with cognitive impairment. Similar techniques have been applied to different populations (e.g., toddlers, students, \&adults) and problem behaviors [6,7].

This procedure was applied based on the rationale that individuals with memory impairment might perform better on difficult memory tasks (low-p) when they first build success by completing easier tasks (high-p). A second objective was to assess the effectiveness of two procedures, one utilizing recognition tasks as high-p items and the other similarly utilizing recall tasks.

\section{METHODS}

\subsection{Informed Consent}

Because participants in this study have some form of dementia, consent was obtained from legal guardians and family members in advance. It was specifically noted that participation was voluntary and subjects could choose to stop at any time. Facility approval and consent was also sought and gained prior to any contact with subjects or family members. Upon meeting with the subject for the first time, the researchers briefly explained the study and procedure in basic terms and received verbal agreement from the subjects to participate. These steps met the requirements for approval of the institution's review board (IRB).

\subsection{Participants}

Participants resided in a Midwestern eldercare facility. Residents suffering from mild to moderate cognitive impairment were referred by staff. Inclusion criteria included mild to moderate cognitive impairment along with the ability to see images and speak to identify images. Exclusion criteria included severe sight and verbal difficulties.

Five residents were referred, with two meeting the inclusion criteria. Each participant was administered the Modified Mini Mental Status Exam to estimate severity of cognitive impairment (3MS) [8]. The 3MS assesses object naming, concentration, immediate/delayed recall, orientation, registration, language, executive functioning, and ability to follow commands. The $3 \mathrm{MS}$ also produces a Mini Mental Status Exam score.

Mabel, a 91-year-old Caucasian female with a high school education, had a primary facility diagnosis of mild dementia and displayed anomia as indicated by an inability to identify common objects after being given the correct answer. Mabel displayed aphasia, referring to key points in a story as "thing," or "it." She repeated stories, often pausing and forgetting which she was speaking about. Mabel's score of 86 on the 3MS (37th percentile for age and education) converted to a MMSE score of 28 .

Sophia, a 92-year-old Caucasian female with an education that included one year of college, had a history of increased memory loss without a facility diagnosis of dementia. During conversation, Sophia demonstrated aphasia and anomia. Aphasia was displayed as difficulty with verbal expression and struggling to finish sentences or stories during conversation. Anomia was indicated by an inability to identify common objects, which is often accompanied by word finding difficulties. Sophia had a $3 \mathrm{MS}$ score of 47 (second percentile for age and education) and an MMSE score of 14 (moderate impairment).

\subsection{Training}

Each research assistant was trained to do data collection through the following procedures: task 
analysis, role-play, and quizzing regarding procedures. The primary data collector facilitated each session while the secondary collector recorded answers, time between tasks, and session length to safeguard procedural integrity. Procedural integrity was calculated for all phases across both participants for correct repetitions of incorrectly identified stimuli during the probability assessment, correct number of stimuli presented during the high probability command sequence (HPCS), and appropriate increases/decreases in intervals during the maintenance phase. Integrity was high across all phases (probability assessment, 96\%; Recognition-to-Recall, 100\%; Recall-to-Recall, 100\%; and maintenance, $97.5 \%)$.

\section{PROCEDURES}

\subsection{Probability Assessment}

A probability assessment was used to empirically determine high-p recognition and recall items and low-p recall (i.e., target) items to be used during subsequent treatment phases. A series of color pictures were shown to the participant who was then asked to identify the object. If the item was a horse, the researcher stated, "This is a type of animal," and then asked, "Can you tell me what type of animal it is?" This procedure was used to assess the participant's ability to engage in the target behavior, recalling the names of objects. Although categorical (e.g., "this is a type of animal") and visual cues were used to assess recall memory during the assessment, this is an established procedure in recall memory research [9].

Participants received feedback after attempting to identify each object. An accurate response was recorded if the first name given for an object was correct and resulted in praise. If the participant answered incorrectly, correct answers were provided. If not correctly identified during the first presentation, the item would be presented a second time at the end of the session to assess participant recall of the object following feedback. This determined the impact of feedback alone.

Ninety-four different images were presented during 16 probability assessment sessions, with each item being presented a minimum of 5 times. Twenty-eight different pictures were presented during each session and were drawn from six categories: animals, clothing, fruit, kitchen items, tools, and vegetables.
High-p items consisted of images correctly identified at least $80 \%$ of the time. Images identified less than $33 \%$ were considered low-p items. Four low-p items were identified for each participant. Low-p items for Mable were avocado, zucchini, broccoli, and squash, while low-p items for Sophia were blender, dates, asparagus, and llama. A total of 70 and 59 high-p items were identified for Mabel and Sophia, respectively.

\subsection{Research Design}

In this intervention, an adaptive alternating treatments design was implemented to determine the effectiveness and magnitude of difference between two high-p/low-p interventions: Recognition-to-Recall and Recall-to-Recall [10]. All intervention sessions were randomized over 12 sessions to guard against carryover effects. Interventions were identical with the exception of the memory task. One low-p recall item and 10 high-p items were presented during each session. Each low-p recall item was asked five times per session over three sessions. Each session consisted of five cycles of memory tasks followed by brief breaks between cycles. Each cycle of memory tasks included a HPCS followed immediately by a low-p recall task. Sessions were held at approximately the same time of day for both participants.

During the Recognition-to-Recall intervention, the participant was required to complete a HPCS involving consecutive and correct identification of three high-p recognition items before the low-p recall task was introduced. Recognition items involved explaining "This is a grouping of four animals (e.g., a cow, horse, deer, and dog). Can you show me which picture is of a (e.g., horse)?" The low-p recall task involved giving the participant a categorical cue (e.g., "This is a type of fruit") and then asking her to identify the item ("Can you tell me what type of fruit this is?").

In Recall-to-Recall, the participant was required to complete a HPCS involving the consecutive and correct identification of three high-p recall items before being asked a low-p recall item. The participant was shown one picture and, following a categorical cue, asked to identify the object. After three successful identifications of high-p recall items, the participant was presented a low$p$ item. The low-p item was always conceptually similar to the last high-p memory task in that conceptually similar items relate to encoding specificity and better learning [4]. For example, if the last high- $p$ target item was a green vegetable 
(e.g., cucumber), the low-p item was also a green vegetable (e.g., asparagus).

The Recognition-to-Recall and Recall-to-Recall intervention sessions were systematically randomized over 12 sessions to guard against carry over effects. One intervention was implemented during each session. One low-p recall item and 10 high-p items were targeted during each session. Each low-p recall item $(\mathrm{N}=4)$ was asked five times per session over three sessions (15 times total). Each session consisted of five cycles of memory tasks followed by one- to two-minute breaks between cycles. The participant and researchers would have a general conversation about the weather, food served during supper, and visits from family during breaks. Sessions were held at approximately the same time of day for both participants (to enhance internal validity). Each cycle of memory tasks included a series of a least three high-p memory tasks followed immediately by a low-p recall task. During the Recognition-to-Recall intervention, the participant was required to consecutively and correctly identify three high-p recognition items before the low-p recall task was introduced. The low-p recall task involved giving the participant a semantic cue (e.g., "This is a type of fruit.") and then asking them to identify the item. Recognition-to-Recall sessions lasted 16 min. 51 $\mathrm{sec}$. on average and ranged $13 \mathrm{~min} .17 \mathrm{sec}$. to 24 min. $40 \mathrm{sec}$.

The Recall-to Recall intervention was similarly structured, incorporating only recall tasks. The participant was required to consecutively and correctly identify three high-p recall items before being asked to recall the name of a low-p target item. After three successful identifications of high-p recall items, the participant was presented with a low-p recall item. On average, Recall-toRecall sessions lasted $11 \mathrm{~min}$. $10 \mathrm{sec}$. and ranged from $8 \mathrm{~min}$. $33 \mathrm{sec}$. to $13 \mathrm{~min} .41 \mathrm{sec}$.

\subsection{Optimal treatment}

Recall-to-Recall was implemented during the optimal treatment phase for both participants. Recall-to-Recall procedures from the alternating treatments phase were replicated during this phase with the exception of using different low-p recall target items. The two low-p items targeted during the Recognition-to-Recall procedure were subsequently targeted using the Recall-to-Recall procedure during this phase.

\subsection{Maintenance}

A Spaced Retrieval (SR) procedure was used to strengthen and maintain gains [11]. In SR sessions, each low-p target item was probed to assess treatment gains. Probes involved using a recall procedure (e.g., "This is a type of fruit. Can you tell me what type of fruit it is?") without feedback. Thereafter, the Recall-to-Recall procedure was implemented on set intervals. Intervals began at two minutes, doubled when the participant correctly identified a low-p target item, and decreased by half when the target item was incorrectly named. A low-p item was considered "mastered" once the participant could recall the name of the item prior to the start of the next SR session. Then the next low-p item was targeted until all four were mastered.

\subsection{Follow-up}

After two months, all four low-p target items were probed using the same procedures from SR sessions. The participant was given a categorical cue (e.g., "This is a type of clothing") and then asked to identify the image ("Can you tell me what type of clothing item it is?").

\section{RESULTS}

Data presented in Figs. 1 and 2 are aggregated in order to better illustrate the magnitude of difference between procedures given each set of stimuli. The probability assessment data point for each participant summarizes the average accuracy of responding for each stimulus set prior to treatment. Each data point in the alternating treatments and optimal treatment phase includes the average percentage of accurate responses for each stimulus in the set as a function of responding per session. For example, the average percentage of accurate responses for avocado and zucchini during sessions one and two for Mabel is represented within the first data point in the Recall-to-Recall phase in Fig. 1. Accurate responding for avocado and zucchini during sessions three and four are presented within the second data point in the Recall-to-Recall phase in Fig. 1 and so on. Average accurate responses during probes prior to $S R$ training is represented by the single data point within SR in Figs. 1 and 2. Follow-up is presented as one data point, which reflects one probe occurring 2-months post-maintenance. Session-by-session data can be located in Tables 1 and 2. With-in session data is available upon request. 


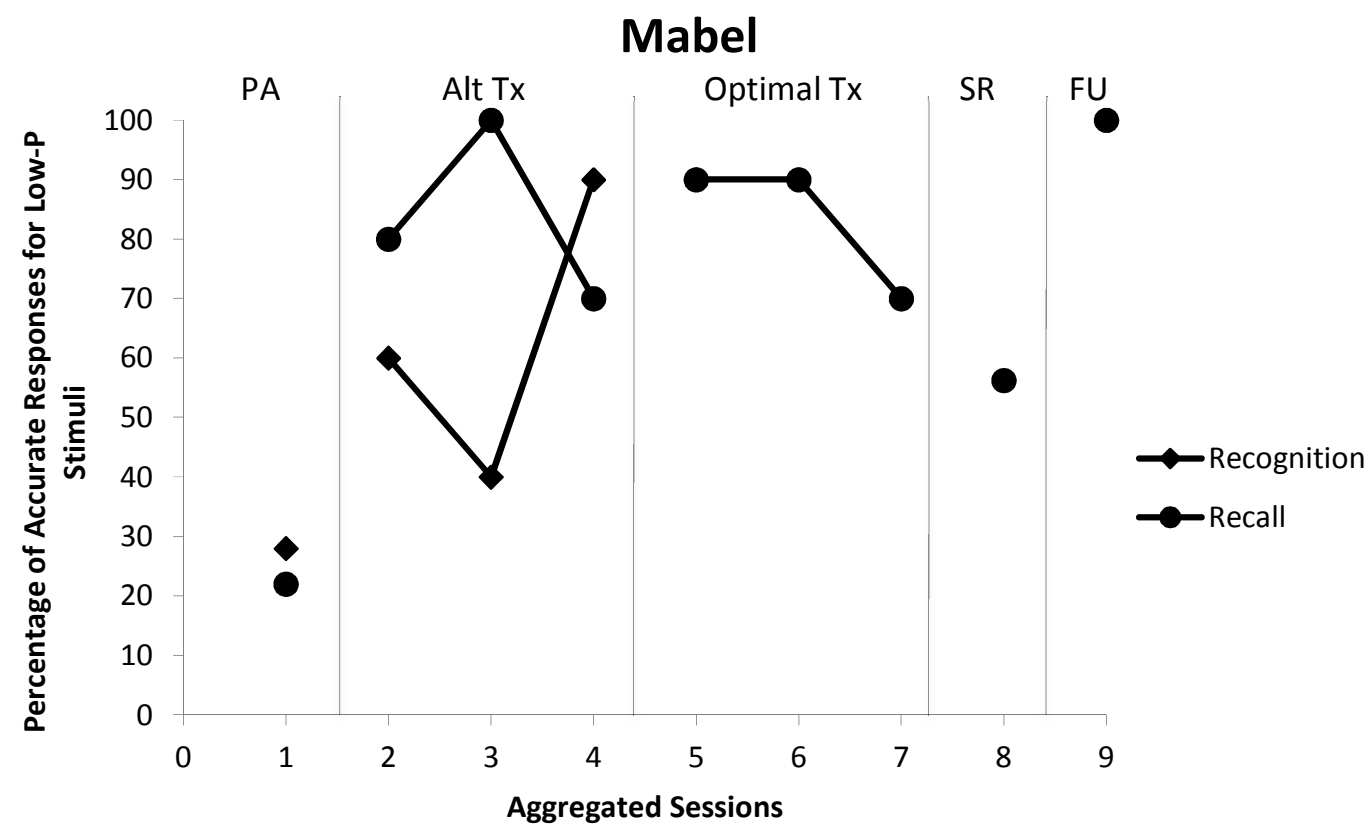

Fig. 1. Aggregated data across phases for Mabel

$P A=$ Probability assessment, Alt Tx = Alternating treatments, $S R=$ Spaced retrieval, $F U=$ Follow-up

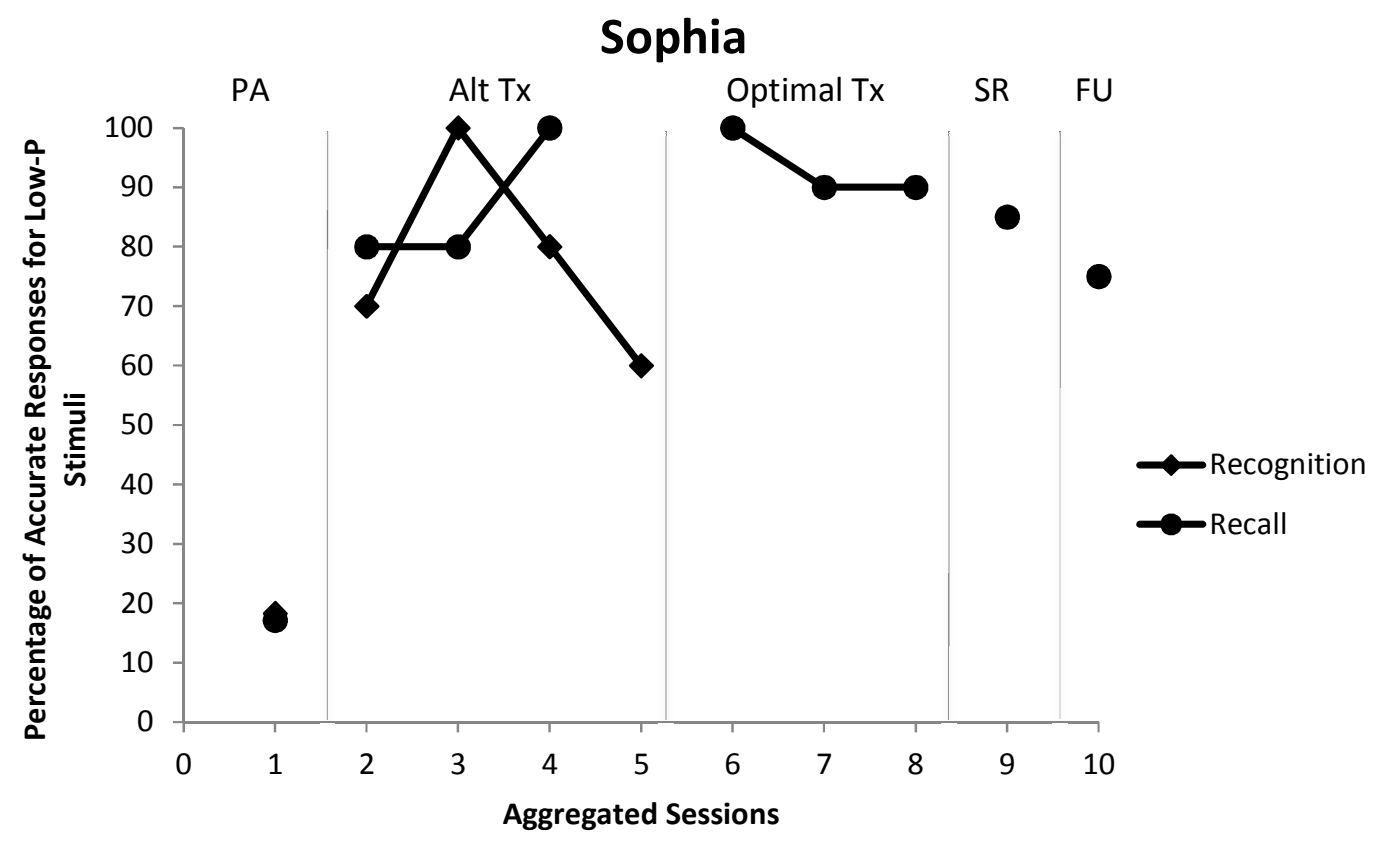

Fig. 2. Aggregated data across phases for Sophia

$P A=$ Probability assessment, $A l t T x=$ Alternating treatments, $S R=$ Spaced retrieval, FU $=$ Follow-up 
Table 1. Mabel: Percentage of correct responses by target Item and phase

\begin{tabular}{lllll}
\hline Phases & \multicolumn{4}{c}{ Low-P target items } \\
\cline { 2 - 5 } & Broccoli & Squash & Avocado & Zucchini \\
\hline Probability assess & 33 & 22 & 33 & 10 \\
Alternating Tx & & & & \\
Session 1 & 60 & 60 & 100 & 60 \\
Session 2 & 60 & 20 & 100 & 100 \\
Session 3 & 100 & 80 & 100 & 60 \\
Optimal Tx & & & & \\
Session 1 & 100 & 80 & - & - \\
Session 2 & 80 & 60 & - & - \\
Session 3 & 100 & 80 & - & 13 \\
SR-probe & 88 & 50 & 75 & 100 \\
Follow-Up & 100 & 100 & 100 & \\
\hline
\end{tabular}

Table 2. Sophia: Percentage of correct responses by target item and phase

\begin{tabular}{lllll}
\hline Phases & \multicolumn{4}{c}{ Low-P target items } \\
\cline { 2 - 5 } & Blender & Dates & Llama & Asparagus \\
\hline Probability assess & 17 & 20 & 14 & 20 \\
Alternating Tx & & & & \\
Session 1 & 60 & 80 & 80 & 80 \\
Session 2 & 100 & 100 & 80 & 80 \\
Session 3 & 80 & 100 & 100 & 100 \\
Session 4 & 60 & - & - & - \\
Optimal Tx & & & & - \\
Session 1 & 100 & 100 & - & - \\
Session 2 & 80 & 100 & - & - \\
Session 3 & 80 & 100 & - & 80 \\
SR-probe & 80 & 100 & 80 & 100 \\
Follow-Up & 0 & 100 & 100 & \\
\hline
\end{tabular}

\subsection{Mabel}

The four low-p target items identified for Mabel were: avocado, zucchini, broccoli, and squash and were recalled with an average accuracy of $33 \%, 10 \%, 33 \%$, and $22 \%$, respectively during the probability assessment. The alternating treatments phase followed the probability assessment. Avocado and zucchini were used during the Recall-Recall intervention. Broccoli and squash were later targeted during Recognition-to-Recall. Results indicated that the Recall-Recall intervention produced $20 \%$ greater accuracy (i.e., 83\% accuracy for RecallRecall vs. $63 \%$ accuracy Recognition-Recall intervention produced; see Fig. 1).

The two items targeted during the Recognitionto-Recall intervention were then targeted using the Recall-to-Recall intervention during the optimal treatment phase. This was to examine if the participant's ability to recall the names of objects would improve once Recall-to-Recall was implemented. Optimal treatment sessions alternately targeted broccoli and squash using sequential randomization. Results indicated that Mabel correctly identified broccoli $93 \%$ of the time compared to $73 \%$ during the RecognitionRecall intervention from the previous phase. Likewise, accuracy for squash increased $20 \%$ with the Recall-Recall intervention $(73 \%$ vs. $53 \%)$.

Fig. 1 also highlights Mabel's ability to recall the names of all target items while probing during spaced retrieval. Mabel identified target items with $56 \%$ accuracy. Over eight days of maintenance, two objects dropped to $34 \%-79 \%$ accuracy: zucchini (67\%) and squash (60\%). Two items became high-p (e.g., $80 \%$ or higher) during SR: avocado (100\%) and broccoli (100\%). Mabel was able to successfully recall the name 
of all target items over the span of 8-min (the initial interval was 2-min) during the first day of SR. Mastery occurred after the first SR session for all items, meaning that Mabel could correctly name the item during a probe at the beginning of the following SR session, which occurred after a period of 24 hours. At a two-month follow-up, Mabel could correctly identify all target items: broccoli, avocado, squash, and zucchini.

\subsection{Sophia}

The four low-p target items identified for Sophia were: blender, dates, llama, and asparagus (recalled with an average accuracy of $17 \%$, $20 \%, 14 \%$, and $20 \%$, respectively during the probability assessment). The two low-p items used during the Recall-Recall intervention (llama and asparagus) were recalled with an average of $17 \%$ accuracy during the probability assessment (see Fig. 2). The two low-p items targeted during Recognition-to-Recall (blender and dates) were recalled with $18 \%$ accuracy during the probability assessment.

Following the probability assessment, the alternating treatments phase was implemented. Llama and asparagus were targeted during the Recall-Recall intervention while blender and dates were targeted during the RecognitionRecall intervention. Sophia recalled llama and asparagus with $87 \%$ accuracy during the Recallto-Recall intervention. Blender and dates were recalled $83 \%$ of the time during Recognition-toRecall. The Recall-Recall intervention was slightly more effective.

Blender and dates were targeted using the Recall-to-Recall intervention during the optimal treatment phase. Sophia was able to correctly identify blender $87 \%$ of the time compared to $75 \%$ during the Recognition-Recall intervention from the previous phase (a 12\% increase). Accuracy for dates increased $7 \%$ once the Recall-Recall intervention was implemented $(100 \%$ vs. $93 \%)$.Accuracy increased for these items by a total of $10 \%$ ( $83 \%$ vs. $93 \%$ ) during the Recall-Recall intervention.

Sophia could correctly identify target items with $85 \%$ accuracy during probes prior to each SR maintenance session. Over five days of SR maintenance all objects became high-p items: blender $(80 \%)$, dates $(100 \%)$, llama $(80 \%)$ and asparagus (80\%). Sophia's intervals increased from 2-min to 8-min during the first day of SR across three items with the exception of dates, which increased to 16-min. Additionally, Sophia mastered all target items after one day of SR. At a two-month follow-up, Sophia could correctly identify three of the four target items: asparagus, dates, and llama (referring to "blender" as "juicer").

\section{DISCUSSION}

The data supported the hypothesis that improvement in recall of low-p items would occur when preceded by a series of high-p items. Both participants' recall of common objects increased when implementing the Recall-to-Recall and Recognition-to-Recall interventions. This finding is noteworthy given the relative paucity of research examining behavioral interventions that target the ability to recall names in persons with cognitive impairment. The data also suggest that the Recall-to-Recall intervention was the most efficient in that it took less time and produced more accurate responses. The data also support the efficacy of studies designed to build upon existing cognitive capabilities to increase the reinforcement level of recall and recognition activities in a procedure easy to apply in a residential setting [12].

It could be argued that enhancement of recall memory could have also been the result of altering an establishing operation. The high-p procedure is designed to enhance the reinforcement associated with memory recall. However, participants could have also had an existing deficit in social feedback, which intensified the participant's inability to identify the names of target items. This uncertainty or selfdoubt was alleviated once the participant was given feedback via social interaction. At this point, accuracy of responses began increasing alongside the participant receiving praise for correct answers. Feedback and praise may have added to the reinforcing effectiveness and increase in frequency of correct responses. In other words, the participants are no longer deprived of social feedback and may find praise reinforcing, which influences their ability to correctly identify the names of common objects. However, feedback alone was not the mechanism of change, as accurate responding did not increase during the probability assessment when feedback was provided.

Secondarily, although the interventions utilized in the current study are novel, they could be explained by the principle of behavioral momentum. The rationale for this study was that 
elderly individuals with cognitive impairment would learn more effectively if they first engage in memory tasks that are relatively easy followed by the presentation of more difficult memory tasks. Presenting the stimuli in this way allowed the participants to contact a high rate of reinforcement, which may build response strength [13]. Momentum may have been gathered from building reinforcer mass and velocity (e.g., consecutive, correct identification of items followed by positive feedback and praise) during the high-p tasks, which influenced the participants' ability to correctly identify low-p items. Also, responding may have been higher in the Recall-to-Recall procedure as memory tasks remained within the same response class (i.e., all recall tasks), whereas the response class differed within the Recognition-to-Recall procedure (i.e., recognition tasks followed by a recall task).

\section{LIMITATIONS}

Support for behavioral momentum procedure was generated in this study. However, the theoretical foundation behind the mechanisms of change of the current procedures is unknown. In other words, it appears as if the intervention works, but it is unclear what psychological principles can account for why it works. The interventions may be effective due to simple rehearsal or may be the effect of a complex system involving rehearsal, feedback, and sequencing of low- and high-p target items. It is also possible that the two subjects chosen for this study were unique in their response to the high-p procedure. There for replication with more subjects is needed.

\section{CONCLUSION}

Future research should address the mechanisms of change involved in both procedures. Future research may also seek to identify how effective this intervention may be for targeting more personally meaningful information such as names of family members. Additional studies also need to determine if this intervention is practical and effective when implemented by lay persons (e.g., nursing assistants).

\section{COMPETING INTERESTS}

Authors have declared that no competing interests exist.

\section{REFERENCES}

1. Alzheimer's Disease International. World Alzheimer's report 2010: The global economic impact of dementia; 2010. Accessed 1 May 2011.

Available:http://www.alz.org/documents/na tional/World Alzheimer Report 2010.pdf

2. Buchanan JA, Christenson A, Houlihan D, Ostrom $\mathrm{C}$. The role of behavior analysis in the rehabilitation of persons with dementia. Beh Ther. 2011;42(1):9-21.

DOI: $10.1016 /$ j.beth.2010.01.003

3. Erber JT. Aging and older adulthood. $2^{\text {nd }}$ ed. Singapore: John Wiley \& Sons, Ltd.; 2010.

4. Reed SK. Cognition: Theories and applications. $8^{\text {th }}$ ed. Belmont, CA: Wadsworth; 2010.

5. Zec RF. Neuropsychological functioning in Alzheimer's disease. In: Parks RW, Zec RF, Wilson RS, editors. Neuropsychology of Alzheimer's disease and other dementias. NY: Oxford University Press; 1993.

6. Belfiore PJ, Pulley-Basile S, Lee DL. Using a high probability command sequence to increase classroom compliance: The role of behavioral momentum. $\mathrm{J}$ of Beh Ed. 2008;17:160-171.

7. Mace FC, Hock ML, Lalli JS, West BJ, Belfiore P, Pinter E, Brown DK. Behavioral momentum in the treatment of noncompliance. $\mathrm{J}$ of Applied Beh Analysis. 1988;21(2):123-141.

8. Teng EL, Chui HC. The modified minimental state (3 MS) examination. J of Clin Psych. 1987;48(8):314-318.

9. Marshall RC, Karow CM, Freed DB, Babock P. Effects of personalized cue form on the learning of subordinate category names by aphasic and non-braindamaged subjects. Aphasiol. 2002;16(7): 763-771.

DOI: $10.1080 / 02687030244000040$

10. Sindelar P, Rosenberg M, Wilson R. An adapted alternating treatments design. Ed and Treat of Children.1985;8:67-86.

11. Camp CJ, Foss JW, O'Hanlon AM, Stevens AB. Memory interventions for persons with dementia. Applied Cog Psych. 1996;10:193-210.

12. Fiksdal BL, Houlihan D, Buchanan JA. Improving recall in a person with dementia: Investigating the effectiveness of memory 
priming and spaced retrieval in an older adult with dementia. Clin Case Studies. 2012;11:393-405.

DOI: $10: 1177 / 1534650112457811$
13. Nevin JA. Response strength in multiple schedules. $J$ of Exp Analysis of Beh. 1974;21(3):389-408.

DOI: $10.1901 /$ jeab.1974.21-389

(c) 2016 Seefeldt et al.; This is an Open Access article distributed under the terms of the Creative Commons Attribution License (http://creativecommons.org/licenses/by/4.0), which permits unrestricted use, distribution, and reproduction in any medium, provided the original work is properly cited.

Peer-review history:

The peer review history for this paper can be accessed here: http://sciencedomain.org/review-history/13828 